\title{
Treatment of Maternal Hypergalactia
}

\author{
Anne Eglash
}

\section{Introduction}

$\mathbf{M}$ ANY BREASTFEEDING MOTHERS struggle with an overabundant milk supply, or hypergalactia, beyond the first few weeks of engorgement. There has been very little research done to define, to explain, or to assist in managing this problem. Lactation specialists have identified several challenges for women with hypergalactia. ${ }^{1}$

Hypergalactia is also termed hyperlactation, oversupply, and engorgement, depending on the literature. The 10th International Classification of Diseases uses the terms hypergalactia, hyperlactation, and increased lactation. The term that is most consistently found in dictionaries to describe excessive milk is hypergalactia.

Because there is not an operational definition with criteria for hypergalactia, the diagnosis would be based on the clinician's impression. A commonsense definition would be the state of producing excessive milk, which leads to discomfort and may compel a nursing mother to express and store milk beyond what the baby is taking, assuming normal infant growth. The caveat of normal infant growth is important because mothers may feel too full at times when the baby is not transferring sufficient milk. An excessive milk supply may appear to be cause for celebration by mothers with insufficient lactation, but mothers with hypergalactia are at increased risk for a fast let-down, ${ }^{2}$ acute mastitis, ${ }^{3}$ plugged ducts, ${ }^{4}$ chronic breast pain, ${ }^{5}$ exclusive pumping, ${ }^{6}$ infant fussiness, ${ }^{7}$ and early weaning. ${ }^{8}$

Many women self-induce hypergalactia by various means. Pumping in addition to nursing stimulates extra milk production. Many herbal supplements are used to increase the milk supply, such as alfalfa, fenugreek, goats rue, fennel, blessed thistle, saw palmetto, and shatavari. ${ }^{9}$ New mothers are often instructed to nurse their babies according to the clock, such as 15-20 minutes on each breast, rather than nursing according to infant feeding cues. This leads some mothers to nurse for longer periods of time than the baby needs to, raising the prolactin level further. ${ }^{7}$

This article is referring to women who persist with hypergalactia despite optimal behavioral interventions to reduce the milk supply.

A mother's milk supply is under extrinsic endocrine control from prolactin and oxytocin. Prolactin is secreted from the anterior pituitary gland in response to nipple stimulation and stimulates milk secretion from lactocytes. The prolactin level is regulated by the frequency and duration of infant suckling or other forms of nipple stimulation such as pumping. Oxytocin release from the posterior pituitary gland occurs in response to several types of sensory input, such as seeing, hearing, smelling, or touching the baby. Myoepithelial cells, which surround alveoli and lactiferous ducts, respond to oxytocin stimulation by contracting to induce milk ejection. Oxytocin release is inhibited by pain and stress. ${ }^{10,11}$

A mother's milk supply is also under local control intrinsically within the breast. Fullness of the breast, within alveoli, is an important factor in controlling milk production. A very full breast is expected to slow milk production, not by simple distension, ${ }^{12}$ but by an increase in the concentration of the "feedback inhibitor of lactation," now known to be nonneural/ peripheral serotonin (5-hydroxytryptamine [5-HT]). ${ }^{12,13}$

There is a paucity of information in the literature describing the percentage of nursing mothers with hypergalactia, whereby these mechanisms of milk production don't achieve a healthy balance of milk production. In addition, there are no human studies to date that have evaluated the underlying etiology for hypergalactia. Many clinicians use behavioral strategies and antilactational substances to help reduce the milk supply.

Maternal symptoms of hypergalactia include breast fullness, an inability to nurse the baby from both breasts for each feeding, and a heavy let-down reflex. The heavy let-down reflex may lead to a shallow latch by the infant, resulting in sore nipples. The mother may also struggle with excessive milk leakage, chronically tender engorged breasts, plugged ducts, and mastitis due to irregular and insufficient breast emptying. Infant symptoms include choking and gasping during the initial let-down, excessive weight gain, fussiness at the breast, excessive flatus, and explosive, green stools. Hypergalactia may be associated with a foremilk-hindmilk imbalance, leading the baby to consume a high proportion of foremilk. High foremilk intake has been suspected in some cases to be associated with blood-streaked infant stools that contain mucus. ${ }^{7}$

Evaluation of hypergalactia could include thyroid function tests to rule out either hyper- or hypothyroidism. In addition, a prolactin level could be measured to see if it is quite elevated. All successfully lactating women should have an elevated prolactin level, so it would be difficult to determine if hypergalactia is truly due to a primary hyperprolactinemia. ${ }^{14}$

The most common behavioral strategy reported to help hypergalactia is block feeding. ${ }^{1,7}$ This involves the mother nursing from one breast for 3-hour blocks ( $\pm 30-60$ minutes).

Department of Family Medicine, University of Wisconsin School of Medicine and Public Health, Mt. Horeb, Wisconsin. 
Table 1. Substances That Reduce the Milk Supply

\begin{tabular}{ll}
\hline Class & \multicolumn{1}{c}{ Compound } \\
\hline Herbs & Sage leaves \\
& Peppermint \\
& Chasteberry \\
& Parsley \\
& Jasmine \\
Pharmaceuticals & Pseudoephedrine \\
& Estrogen \\
& Carbergoline \\
& Bromocriptine \\
\hline
\end{tabular}

For example, the mother nurses from the right breast from noon to 3 o'clock. Then from 3 to 6 o'clock all nursing comes from the left breast. This allows one breast to remain undisturbed for 3 hours or more at one time, so that local control from an increase in 5-HT will provide feedback to lactocytes to decrease the milk supply. Usually within 24-48 hours the mother notices a significant decline in her milk supply. Occasionally the supply will drop so much that mom will need to nurse from both sides for each feeding.

If block feeding is not successful, the next step would be to try various supplements or medications to reduce the milk supply (Table 1). Common substances include herbs, homeopathy, pseudoephedrine, estrogen in the form of the combined birth control pill, and, finally, antiprolactin medications.

Sometimes these substances are needed to help a woman wean because the normal mechanism of breast fullness results in complications such as plugged ducts and mastitis, making it difficult to fully wean.

\section{Herbs}

A few herbs are clinically useful to reduce the milk supply. Sage, or Salvia officinalis, is the most common herb used to reduce milk supply. Sage tea or extract made from the leaves is typically recommended, although there are no studies on the use of sage for hypergalactia and very few on its effect on the nursing baby. ${ }^{15}$ Sage tea may be prepared by steeping 1$3 \mathrm{~g}$ of dried sage leaves in a cup of hot water. ${ }^{16}$ There are several commercial preparations of sage extract available.

The mother should be advised to just use one dose of the extract (as recommended by the manufacturer) or 1 cup of tea and to observe the effect on her supply, as well as any behavioral change in the baby, over the next several hours. If she does not notice a difference in supply in 8-12 hours, then she can try another, stronger dose. Once she sees a response, she should just use it as needed. Often women will use one dose every 12 hours for 3 days to keep their supply down.

Sage is known to have several side effects in high doses, including nausea, vomiting, and dizziness. It can induce wheezing, lower the blood sugar, and induce seizures, so high doses should be avoided in asthmatics, diabetics, and people prone to seizures. It is considered safe when used as a food. ${ }^{15,16}$

Jasmine flowers applied topically and chasteberry taken orally are both suspected to reduce the prolactin level and may have an effect on reducing milk production. Very little research exists to guide clinical use of these herbs. ${ }^{17}$

Parsley is believed to reduce the prolactin level and could reduce the milk supply when eaten as a food, such as in tabouli. ${ }^{18}$
Peppermint oil might reduce the milk supply when used topically. Peppermint in high doses can be toxic and should be kept away from the baby. ${ }^{19}$

\section{Homeopathy}

Homeopathic medications are based on the "Law of Similars," or "like curing like." The principle is that substances given in low doses may be effective for symptoms that are produced when the substance is ingested in high doses. ${ }^{20}$ Potency of homeopathic medications are determined by the dilution of the substance. For example, a 30C dilution indicates that the substance has been diluted to $10^{-60}$. Homeopathic medications found to be helpful in reducing the milk supply include lac caninum 30C, Pulsatilla 30C, and Ricinus communis 30C. These are purchased as small pellets. The typical dose is five pellets sublingually two to three times a day, until a decrease in milk supply is noticed. The medication is then used two to three times a day as needed to keep the milk supply down. There are minimal to no side effects noted with homeopathic agents, other than gastrointestinal intolerance to the lactose that is added to the pellets.

\section{Pharmaceuticals}

Pseudoephedrine is a commonly used decongestant that has been found to decrease the milk supply. One study found that a 60-mg dose of pseudoephedrine was associated with a $24 \%$ decrease in milk production. It is unclear if the effect is achieved by decreasing the prolactin level. ${ }^{21}$ Pseudoephedrine could first be given as a 30-mg dose, watching for side effects of jitteriness, irritability, and insomnia. If the $30-\mathrm{mg}$ dose does not decrease the supply in 8-12 hours and it is tolerated well, the mother could increase the dose to $60 \mathrm{mg}$. Once the mother notices a drop in her milk supply, she can use it every 12 hours as needed to keep her supply down to a manageable level. It is important to not prescribe this for a set duration of time, such as twice a day for 3 days, as this may drop the supply too low. By using it just as needed, the mother can more carefully determine her body's response to the medication.

Estrogen has a negative effect on lactation, reducing the milk supply. ${ }^{10,22,23}$ Estrogen can be given as the combined birth control pill, once a day for a week, and then stopped. The mother should see a decline in her milk supply by 5-7 days. If her supply rises over time, she can be treated again short term with the combined birth control pill.

Treatment with estrogen increases the risk of thromboembolism in the mother, especially if prescribed before 4 weeks postpartum. $^{23}$

If none of the preceding treatments reduces the milk supply, the final step would be to use an antiprolactin medication such as bromocriptine or cabergoline. Both are effective in reducing the milk supply early postpartum. Cabergoline has been shown to have fewer side effects than bromocriptine. ${ }^{24}$ However, very little is known about transmission of cabergoline into breastmilk, whereas very little bromocriptine is transferred into breastmilk. ${ }^{25}$

There are no published reports on the effectiveness of either of these medications in the treatment of hypergalactia during later stages of lactation.

Because of cabergoline's lower side effect profile, I have chosen to use cabergoline 0.25 twice a day for 1 day as the last resort in nursing mothers with hypergalactia who have 
not responded to other treatments, particularly for mothers who are attempting to wean without success. Cabergoline is long acting, with a half-life of 63-69 hours. ${ }^{26}$ For that reason, I advise mothers to express and discard their breastmilk for approximately 5 days after using cabergoline.

\section{Conclusions}

Women with true hypergalactia are at risk for medical complications of breastfeeding, including maternal pain and infections, as well as infant symptoms of gastrointestinal distress. These mothers often have difficulty weaning. There are several strategies that can be used to help lower the milk supply, including changes to the feeding strategy, complementary medications, and pharmaceuticals. Mothers should be followed over time to properly management side effects and outcomes of treatment.

\section{Disclosure Statement}

No competing financial interests exist.

\section{References}

1. Wilson-Clay B. Milk oversupply. J Hum Lact 2006;22: 218-220.

2. Prime DK, Kent JC, Hepworth AR, et al. Dynamics of milk removal during simultaneous breast expression in women. Breastfeed Med 2012;7:100-106.

3. Riordan JM, Nichols FH. A descriptive study of lactation mastitis in long-term breastfeeding women. J Hum Lact 1990;6:53-58.

4. Campbell SH. Recurrent plugged ducts. J Hum Lact 2006; 22:340-343.

5. Witt A, Mason MJ, Burgess K, et al. A case control study of bacterial species and colony count in milk of breastfeeding women with chronic pain. Breastfeed Med 2014;9:29-34.

6. Clemons SN, Amir LH. Breastfeeding women's experience of expressing: A descriptive study. J Hum Lact 2010;26:258-265.

7. Smillie CM, Hetzel-Campbell S, Iwinski S. Hyperlactation: How left-brained 'rules' for breastfeeding can wreak havoc with a natural process. Newborn Infant Nurs Rev 2005;5:49-58.

8. Humenick S, Hill P. Breast engorgement: Patterns and selected outcomes. J Hum Lact 1994;10:87-93.

9. Galactagogues to Increase Milk Production. Available at www.lowmilksupply.org/herbalgalactagogues.shtml (accessed September 5, 2014).

10. Stuebe AM. Enabling women to achieve their breastfeeding goals. Obstet Gynecol 2014;123:643-652.

11. Czank C, Henderson JJ, Kent JC, et al. Hormonal control of the lactation cycle. In: Hale T, Hartmann P, eds. Hale \& Hartmann's Textbook of Human Lactation. Hale Publishing, Amarillo, TX, 2007, pp. 89-111.

12. Stull MA, Pai V, Vomachka AJ, et al. Mammary gland homeostasis employs serotonergic regulation of epithelial tight junctions. Proc Natl Acad Sci U S A 2007;104:1670816713.

13. Hernandez LL, Stiening CM, Wheelock JB, et al. Evaluation of serotonin as a feedback inhibitor of lactation in the bovine. J Dairy Sci 2008;91:1834-1844.

14. Adamopoulos DA, Kapolla N. Prolactin concentration in milk and plasma of puerperal women and patients with galactorrhea. J Endocrinol Invest 1984;7:273-276.

15. LactMed Database. Sage. Available at http://toxnet.nlm .nih.gov/cgi-bin/sis/search2/f?./temp/ V9u6ic:1- (accessed September 5, 2014).

16. Sage. Available at www.consumerlab.com/tnp.asp?chunkiid $=$ 111802- (accessed September 6, 2014).

17. Budzynska K, Gardner ZE, Dugoua JJ, et al. Systematic review of breastfeeding and herbs. Breastfeed Med 2012; 7:489-503.

18. Schaefer C. Drugs During Pregnancy and Lactation, 2nd ed. Elsevier BV, Amsterdam, 2007, pp. 4, 13.

19. Conover E, Buehler BA. Use of herbal agents by breastfeeding women may affect infants. Pediatr Ann 2004; 33:235-240.

20. Pizzorno JE, Murray MT. Textbook of Natural Medicine, 4th ed. Churchill Livingstone, Elsevier, London, 2013, pp. 314-326.

21. Aljazaf K, Hale TW, Ilett KF, et al. Pseudoephedrine: Effects on milk production in women and estimation of infant exposure via breastmilk. Br J Clin Pharmacol 2003; $56: 18-24$.

22. Creasy RK, Resnik R, Iams JD, et al. Creasy and Resnik's Maternal-Fetal Medicine: Principles and Practice, 7th ed. Saunders, Philadelphia, 2014, pp. 9, 12-130.

23. LactMed Database. Combined Oral Contraceptives. Available at http://toxnet.nlm.nih.gov/cgi-bin/sis/search2/ f?./temp/ 92Bfx1:1- (accessed September 6, 2014).

24. Aydin Y, Atis A, Kaleliq S, et al. Carbergoline versus bromocriptine for symptomatic treatment of premenstrual mastalgia: A randomized, open-label study. Eur J Obstet Gynecol Reprod Biol 2010;150:203-206.

25. TOXNET Database. Bromocriptine. Available at http:// toxnet.nlm.nih.gov/cgi-bin/sis/search2/f?./temp/ 9MiljI:1 (accessed September 7, 2014).

26. Cabergoline. Available at https://online.epocrates.com/ noFrame/showPage $?$ method $=$ drugs \&MonographId $=659 \&$ ActiveSectionId =7 (accessed September 7, 2014).

Address correspondence to:

Anne Eglash, MD, FABM Department of Family Medicine University of Wisconsin School of Medicine and Public Health 600 North 8th Street Mt. Horeb, WI 53572

E-mail: areglash@wisc.edu 